\title{
ANÁLISIS CRÍTICO DE LA FORMACIÓN Y EJERCICIO DE LA ABOGACÍA EN EL ECUADOR
}

\author{
MENESES SOTOMAYOR, María Cristina ${ }^{66}$.
}

SUMARIO: 1. Introducción. - 2. El libre desarrollo de la personalidad y la dignidad humana en el Ecuador. - 3. Vocación Profesional y Abogacía. - 4. Formación profesional y Estado.

\section{Introducción}

¿Es la sobreoferta de profesionales de abogacía, una justa causa para limitar la libertad de escoger profesión, en base a la vocación (aptitudes intelectuales, éticas y físicas), limitando nuevos ingresos en Escuelas de Derecho a nivel nacional y emprender en campañas publicitarias ${ }^{67}$ que pretenden desvalorizar una de las profesiones más tradicionales y nobles de la humanidad?

¿O será acaso que en un trabajo conjunto estatal y privado, se debe motivar a los nuevos profesionales al perfeccionamiento, entendido como un proceso de conocimientos integrales a través de las especializaciones y posgrados que les permita hacer frente a las demandas laborales con una mayor autonomía y efectividad?

El presente artículo está orientado a analizar aspectos básicos como el derecho al libre desarrollo de la personalidad, la vocación, el derecho y la abogacía en las pasadas, presentes y futuras generaciones, el abogado como defensor y mediador al servicio de la comunidad, generador de conocimiento y nuevas interpretaciones que contribuyan la realización de los ideales constitucionales de libertad y justicia.

\section{CONTENIDO.-}

\section{El libre desarrollo de la personalidad y la dignidad humana en el Ecuador}

La Constitución vigente declara al Ecuador como un estado constitucional DE derechos y justicia o como también se le puede llamar Estado garantista; es decir, se cimienta y construye sobre los derechos fundamentales de la persona; y sobre aquel que ha sido considerado fundamento de todos ellos, la dignidad humana; la Carta magna, al igual que la mayoría de las constituciones latinoamericanas, así lo reconocen; en la nuestra expresamente se manifiesta en su artículo 84: "La Asamblea Nacional y todo órgano con potestad normativa tendrá la obligación de adecuar, formal y materialmente, las leyes y demás normas jurídicas a los derechos previstos en la Constitución y los tratados

${ }^{66}$ Licenciada en ciencias sociales, económicas y políticas. Abogada. Doctora en Jurisprudencia. Diplomado en Práctica Procesal, Investigativa y Oralidad. Magister en Ciencias Penales. Magister en Derecho Administrativo. Estudiante de Doctorado en Derecho y Ciencias Políticas (Universidad Nacional de Piura). Directora y docente de la Escuela de Derecho de la Universidad Internacional del Ecuador sede Loja.

${ }^{67}$ http://www.youtube.com/watch?v=MgTOL3VKPCI 
internacionales, y los que sean necesarios para garantizar la dignidad del ser humano o de las comunidades, pueblos y nacionalidades. En ningún caso, la reforma de la Constitución, las leyes, otras normas jurídicas ni los actos del poder público atentarán contra los derechos que reconoce la Constitución".

Hablar de dignidad humana, es hablar de ese rasgo característico, único de los seres humanos, que le dota de capacidad de autodeterminación y de realización del libre desarrollo de la personalidad.

Tal como afirma nuestra Constitución, la dignidad humana se constituye en sustento inmejorable en el ejercicio de los derechos fundamentales, es más, en sus artículos 7 y 8 , afianza este postulado con la cláusula abierta de los derechos humanos, que implica el reconocimiento de los derechos y garantías establecidos en la norma suprema y en los instrumentos internacionales de derechos humanos ratificados por el Ecuador, sin excluir los demás derechos que se deriven de la dignidad de las personas y los pueblos y que son necesarios para su pleno desenvolvimiento; y, el principio de progresividad de derechos, determinando que los mismos deben ser desarrollados paulatinamente a fin de extender su ámbito de protección. Obligando al Estado ecuatoriano a generar las condiciones necesarias para su pleno reconocimiento y ejercicio. El contenido de los derechos debe ser desarrollado doctrinario, normativo, jurisprudencialmente y a través de políticas públicas.

El derecho al libre desarrollo de la personalidad, sin más limitaciones que los derechos de los demás, se encuentra previsto en la Constitución ecuatoriana, numeral 5, artículo 66, dentro de los derechos de libertad; estableciendo la obligatoriedad estatal de reconocer la facultad natural de toda persona a decidir sobre su propia vida, siguiendo su vocación profesional, misma que inicia en el primer día de ingreso al sistema educativo ecuatoriano, sin imposiciones, controles o impedimentos por parte de ninguna persona natural o jurídica. Éste derecho comprende: a) la facultad de hacer o no hacer de acuerdo a su voluntad, buscando la realización de las metas propias de cada persona, en base a su vocación y carácter, la posibilidad de optar por una profesión u oficio, lógicamente sin afectar el derecho ajeno o vulnerar la legislación vigente; $\mathrm{y}, b$ ) la prohibición que el Estado imponga decisiones o impida acciones al titular del derecho, que es el ser humano. (Para abundar en éste tema revisar sentencia Nro. C-355-06. Corte Constitucional de Colombia) ${ }^{68}$.

El sistema interamericano de derechos humanos, en los últimos años, incorpora a su jurisprudencia el proyecto de vida, como un derecho a la realización personal de acuerdo a la vocación y pretensión personal, permitiéndonos ser actores de nuestra propia vida,

68 http://www.corteconstitucional.gov.co/relatoria/2006/C-355-06.htm: “...La primera y más importante de todas la consecuencias del derecho al libre desarrollo de la personalidad y autonomía, consiste en que los asuntos que sólo a la persona atañen, sólo por ella deben ser decididos. Decidir por ella es arrebatarle su condición ética, reducirla a su condición de objeto, cosificarla, convertirla en medio para los fines que por fuera de ella se eligen. Cuando el Estado resuelve reconocer la autonomía de la persona, lo que ha decidido, es constatar el ámbito que le corresponde como sujeto ético: dejarla que decida sobre su propia vida, sobre lo bueno y lo malo, sobre el sentido de su existencia..." "...El derecho al libre desarrollo de la personalidad no es un simple derecho, es un principio genérico y omnicomprensivo cuya finalidad es cobijar aquellos aspectos de la autodeterminación del individuo, no garantizados en forma especial por otros derechos, de tal manera que la persona goce de una protección constitucional para tomar, sin intromisiones ni presiones las decisiones que estime importantes en su propia vida. La primera consecuencia que se deriva de la autonomía consiste en que es la propia persona quien debe darle sentido a su existencia y en armonía con ésta, un rumbo...." 
encaminando nuestra existencia y opciones profesionales, de acuerdo a nuestros intereses, aptitudes y capacidades físicas e intelectuales.

En éste contexto, somos libres de escoger la (s) profesión (es) u oficio(s) acordes a nuestra vocación, enmarcadas en el respeto al derecho ajeno y legislación nacional vigente.

\section{Vocación profesional y abogacía}

Vocación proviene del término latino "vocatio" que significa convocatoria, llamado, invitación; es decir, la convocatoria o inclinación, que basado en habilidades específicas, las capacidades, anhelos nos permite escoger carrera, profesión, oficio o estado.

El Dr. Hermán Jaramillo Ordóñez, jurista lojano y catedrático de la UIDE extensión Loja, al respecto, manifiesta: "La vocación profesional es un proyecto de intenciones y aspiraciones que desea alcanzar una persona, al inclinarse, prepararse y formarse con deseos de superación, para el ejercicio de una profesión, con espíritu de servicio, tomando en cuenta lo que conoce, lo que piensa y desea hasta lograr los objetivos y metas trazadas."... "Es el resultado de combinar nuestras aptitudes y potencialidades con los valores éticos y jurídicos que desea la comunidad" (JARAMILLO, 2012, p. 15$)^{69}$.

Algunas discusiones se han generado respecto a si la vocación nace o se hace; múltiples y variadas han sido las respuestas, presentándose un elemento común en todas ellas, el autoconocimiento, y es que la elección acertada de profesión depende del conocimiento que el adolescente tenga de sí mismo, su valoración, aceptación e identificación de sus rasgos. A éste autoconocimiento se suma el apoyo incondicional y orientación, en primer término de la familia, posterior de docentes y formación recibida en la escuela y colegio. De ésta forma, se evidencia la responsabilidad compartida, entre familia y estado, a través de una formación integral, orientación y educación de calidad que debe impartirse desde la instrucción primaria y secundaria.

Para abundar en el tema, hago mías las expresiones que de Johann Fichte, se expusieron en una de las ponencias presentadas en el Congreso: Retos y Expectativas de la Universidad, realizado en junio del año 2006, en Puebla México, en torno a la vocación ${ }^{70}$ :

"Pero debo abrir mis ojos, debo aprender a través del conocimiento de mí mismo: necesito saber lo que me constriñe. Esa es mi vocación primera. Entonces, bajo ese antecedente debo construir mi propio modo de pensar. Así seré absolutamente independiente, y proveeré y perfeccionaré mis propios actos y mis obras. La fuente de todo lo demás ya no le será extraño a lo más íntimo de mi espíritu. Ya soy enteramente mi propia creación y puedo seguir confiadamente la senda de mi espíritu. Ya no soy el producto de la Naturaleza sino de mí mismo. He llegado a aceptar mi propia obscuridad y mis incertidumbres, con la libertad de haberme resuelto simplemente a hacerlo. He escogido el sistema en el que mis propósitos se han fijado un destino, porque he reconocido en ello consistencia con mi dignidad y mi vocación. Mi libertad y mi convicción han regresado al punto en el que la Naturaleza me había dejado. Acepto lo que ella ahora me depara; -pero no lo acepto porque así debo hacerlo, sino porque así lo creo, así lo he decidido".

69 JARAMILlo ORDÓÑEZ, H. (2012). Ética Jurídica. (Primera edición, pp 15- 16) Loja: Offsset Grafimundo: “... la vocación requiere de aptitudes intelectuales, éticas y fisicas cuya posesión es una ventaja indudable para adquirir el éxito..."

${ }^{70}$ http://www.congresoretosyexpectativas.udg.mx/Congreso\%206/Eje\%202/Ponencia_338.pdf 
El plan nacional del buen vivir, determina que "Ecuador es uno de los países latinoamericanos con menor cobertura de educación superior, es, entonces, una prioridad aumentar el acceso a este nivel educativo. A la par, se debe garantizar igualdad de oportunidades para todas y todos..." "De igual manera, deberá ser política pública la inversión en talentos humanos que estudien prioritariamente en áreas específicas ligadas a las necesidades de desarrollo del país, a través de becas para estudios de postgrado en universidades de primer nivel." Se expresa además el afán del Estado por vincular "la investigación producida en las universidades a los institutos públicos de investigación a fin de crear sinergias que permitan aportar valor agregado a la industria nacional..." Es evidente el interés estatal por brindar una educación de calidad, en igualdad de oportunidades, apoyando la vocación profesional de los ecuatorianos, inclusive a través de becas, sin proscribir carreras, sino al contrario plantea enfoques de aplicación, en base a las nuevas necesidades estatales, por ejemplo en el área de derecho aplicadas a litigios ambientales, circunscripciones territoriales originarias, ejercicio ético del desarrollo biotecnológico, legislación para la investigación de la biotecnología y sus implicaciones jurídicas, sistemas de patentes internacionales que requieren la protección de nuestra soberanía natural e intelectual como la biópolis planteada por el plan nacional del buen vivir; y, además en el caso de la transmisión y consumo de energías alternativas limpias y eficientes, existe un vasto campo novel referido a la legislación que norme el espectro radioeléctrico y las afectaciones ambientales por mínimas que sean de la generación eólica, solar y de biocombustibles.

El plan del buen vivir es concebido, consciente de la necesidad de trabajo interdisciplinario, así como también de la imposibilidad de intentar cambiar el interés de carreras tradicionales y con aceptación social, pues primeramente sería necesario un planteamiento coherente vocacional desde el primer año de educación básica, hasta el tercer nivel de educación, esto considerando que a ningún ciudadano se le podría restringir que opte por la profesión que considera como vocación. Si éste fuera el interés, deberían existir pasos previos, que al momento no los hay.

Referente a la vocación profesional del abogado, debemos partir por conocer ¿Quién es un abogado? ... Como muchos autores lo han dicho, el abogado es el amante de la paz, dedicado moral, ética y académicamente a la defensa y asesoramiento de las partes, en aras de mantener la convivencia pacífica y seguridad jurídica.

Si nos remitimos, al origen del abogado, desde el substrato sobre el que se fundamenta nuestro derecho, debemos obligadamente mirar a Grecia y Roma.

En Grecia, la abogacía era ejercida por los ciudadanos libres y selectos, pues se decía que era trabajo de nobles y ciudadanos íntegros.

Bueno es recordar, en nuestras primeras clases de derecho romano, aquella clasificación de los abogados: Los advocati o causadaci (aquellos que asistían a otros en juicio); y, los iurisconsulti o iurisprudenti (que aconsejaban a los jueces, abogados y a las partes). La diferencia, según Cicerón, los jurisconsultos, pertenecían a la clase privilegiada y conocían a fondo el derecho; mientras que los abogados, eran producto de un empirismo ciudadano fruto de la experiencia social y no de la instrucción. Ésta era la clasificación que nos presentaba el mundo romana en su etapa clásica. 
Ya, en el siglo XII, surge una verdadera ciencia del Derecho, iniciada por la Escuela de glosadores, creadores de la primera escuela de juristas ${ }^{71}$. Bologna, la primera universidad, en materia jurídica, estuvo enfocado en el estudio del digesto, decreto de graciano, las instituciones y el código, bien conocido además que su número de estudiantes fue muy reducido.

Como herederos del Derecho castellano, los primeros abogados, llegan a tierras americanas procedentes de España. Ya en América, la educación universitaria, incluida la jurídica, estaba reservada a las familias de origen español, hijos de funcionarios coloniales, aceptados bajo juramento en base a antecedentes familiares; siguiendo la tradición de las siete partidas estudios negados a mujeres, locos, desmemoriados, ciegos, sordos, pródigos, enjuiciados por adulterio, traición, homicidio, u otro delito de gravedad semejante, los herejes, sus hijos y nietos.

Bien es conocido, que a lo largo de la historia, en diferentes épocas, ya se pretendió hablar de la existencia numerosa de abogados, según los historiadores, la causa era la preocupación por los daños que ellos podían producir, como iniciadores de problemas y litigios.

La independencia americana, inicialmente en materia universitaria, afectó principalmente en la participación estudiantil, presentándose muchos desertores; situación que fue regulada con el paso de los años e inclusive garantizada en sus cartas magnas como un derecho fundamental.

Muchas fueron las batallas libradas, para que la educación sea un derecho general al que acceda cualquier ciudadano, no solamente las élites y clases pudientes; jornadas de trabajo y valentía, se evidenciaron también para que las mujeres puedan acceder a la educación superior, sirviendo a su comunidad con la misma capacidad y conocimiento que los caballeros.

Hombres y mujeres que a lo largo de la historia defendieron sus derechos y nos heredaron libertad, que sólo se consigue a través del conocimiento e igualdad, pues la educación es un derecho de todos.

Muestra de que las conquistas sociales respecto al tema de los derechos tiene que ver con la evolución histórica y cultural, se destaca la figura de Obdulia Romelia Luna Luna, primera mujer abogada en el país, que abre camino a la equidad de acceso a la formación jurídica a las mujeres en el año 1928, evento destacable que hoy, quienes ejercemos la abogacía consideramos una demostración fiel de que la dedicación individual hacia la consecución de conquistas colectivas por los derechos en nuestro país se hace efectiva a luz de la justicia entendida de manera genérica.

El abogado es el constructor de ideas, defensor y mediador al servicio de la comunidad, generador de conocimiento y nuevas interpretaciones que contribuyen la realización de los ideales constitucionales de libertad y justicia.

71 ReIG, José y LARreA, Juan (2000). Manual de Historia del Derecho en el Ecuador. Quito: Corporación de Estudios y Publicaciones. 
Es innegable, que la corrupción y falta de ética profesional, ha tomado algunas conciencias, no solamente en nuestra profesión, sino en algunas otras profesiones u oficios; es precisamente esa la batalla por ganar. Debemos enfocar nuestro trabajo de manera tal, que sean las presentes y futuras generaciones, quienes con voz fuerte y mano firme, sienten bases de la transparencia y honestidad de los ecuatorianos en su vida profesional.

\section{Formación profesional y estado}

La constitución de la República garantiza nuestro derecho a la educación en la siguiente forma:

- El artículo 3, determina como deber primordial del Estado: “... 1. Garantizar sin discriminación alguna el efectivo goce de los derechos establecidos en la Constitución y en los instrumentos internacionales, en particular la educación, la salud, la alimentación, la seguridad social y el agua para sus habitantes...."

- Artículo 26: "La educación es un derecho de las personas a lo largo de su vida y un deber ineludible e inexcusable del Estado. Constituye un área prioritaria de la política pública y de la inversión estatal, garantía de la igualdad e inclusión social y condición indispensable para el buen vivir. Las personas, las familias y la sociedad tienen el derecho y la responsabilidad de participar en el proceso educativo."

- Artículo 350: El sistema de educación superior tiene como finalidad la formación académica y profesional con visión científica y humanista; la investigación científica y tecnológica; la innovación, promoción, desarrollo y difusión de los saberes y las culturas; la construcción de soluciones para los problemas del país, en relación con los objetivos del régimen de desarrollo.

De la misma forma, la Ley Orgánica de Educación superior, establece:

Art. 3.- Fines de la Educación Superior.- La educación superior de carácter humanista, cultural y científica constituye un derecho de las personas y un bien público social que, de conformidad con la Constitución de la República, responderá al interés público y no estará al servicio de intereses individuales y corporativos.

Art. 4.- Derecho a la Educación Superior.- El derecho a la educación superior consiste en el ejercicio efectivo de la igualdad de oportunidades, en función de los méritos respectivos, a fin de acceder a una formación académica y profesional con producción de conocimiento pertinente y de excelencia.

Las ciudadanas y los ciudadanos en forma individual y colectiva, las comunidades, pueblos y nacionalidades tienen el derecho y la responsabilidad de participar en el proceso educativo superior, a través de los mecanismos establecidos en la Constitución y esta Ley.

Art. 5.- Derechos de las y los estudiantes.- Son derechos de las y los estudiantes los siguientes:

a) Acceder, movilizarse, permanecer, egresar y titularse sin discriminación conforme sus méritos académicos;

b) Acceder a una educación superior de calidad y pertinente, que permita iniciar una carrera académica y/o profesional en igualdad de oportunidades; 
c) Contar y acceder a los medios y recursos adecuados para su formación superior; garantizados por la Constitución;

d) Participar en el proceso de evaluación y acreditación de su carrera;

e) Elegir y ser elegido para las representaciones estudiantiles e integrar el cogobierno, en el caso de las universidades y escuelas politécnicas;

f) Ejercer la libertad de asociarse, expresarse y completar su formación bajo la más amplia libertad de cátedra e investigativa;

g) Participar en el proceso de construcción, difusión y aplicación del conocimiento;

h) El derecho a recibir una educación superior laica, intercultural, democrática, incluyente y diversa, que impulse la equidad de género, la justicia y la paz; e,

i) Obtener de acuerdo con sus méritos académicos becas, créditos y otras formas de apoyo económico que le garantice igualdad de oportunidades en el proceso de formación de educación superior.

De la revisión previa de nuestra legislación, se puede afirmar categóricamente el deber del Estado de garantizar y efectivizar el derecho a la educación respetando los derechos fundamentales establecidos en la Constitución de la República, vale la pena recordar lo manifestado por el doctrinario Manuel Atienza: "Tener un derecho fundamental tiene que, significar que, al menos en principio, ninguna directriz política ni objetivo social puede prevalecer frente a él. El que el ejercicio de un derecho implique un obstáculo para llevar a cabo una determinada política gubernamental o que, incluso, sitúe al Gobierno ante un auténtico dilema no puede ser por sí misma una razón, válida para limitar dicho Derecho."72 (ATIENZA, 2012, p 134), por lo tanto el respeto al libre desarrollo de la personalidad, analizado en líneas anteriores, que otorga la facultad de hacer o no hacer de acuerdo a su voluntad, buscando la realización de las metas propias de cada persona, en base a su vocación y carácter, la posibilidad de optar por una profesión u oficio, lógicamente sin afectar el derecho ajeno o vulnerar la normativa vigente, debe ser respetado y apoyado por el Estado y la sociedad.

Creo firmemente, en la necesidad de intervención del Estado, a través de sus organismos competentes, para garantizar la calidad de la educación superior, muy ligada al desempeño profesional; y, es que solamente el trabajo mancomunado de Estado, Universidad y sociedad, permitirá el desarrollo y crecimiento del país, de cada ecuatoriano.

Pero la intervención, no puede ni debe darse únicamente a nivel superior, la educación es un todo, educación integral, que parte desde la primera escuela, hogar, pero afianzada en la instrucción primaria y secundaria, meditemos en ésta reflexión de Maria Montessori: " $E l$ niño, con su enorme potencial físico e intelectual, es un milagro frente a nosotros. Este hecho debe ser transmitido a todos los padres, educadores y personas interesadas en niños, porque la educación desde el comienzo de la vida podría cambiar verdaderamente el presente y futuro de la sociedad. Tenemos que tener claro, eso sí, que el desarrollo del potencial humano no está determinado por nosotros. Solo podemos servir al desarrollo del niño, pues este se realiza en un espacio en el que hay leyes que rigen el funcionamiento de cada ser humano y cada desarrollo tiene que estar en armonía con todo el mundo que nos rodea y con todo el universo".

Las falencias que hoy en día se pueden detectar en la vida universitaria e inclusive profesional, están relacionadas con los conocimientos adquiridos en edades tempranas y

\footnotetext{
${ }^{72}$ AtIEnZA, M (2012). Tras la Justicia. Barcelona: Editorial Planeta S.A.
} 
fortalecidas en etapas juveniles. Y es, precisamente ahí, en donde también necesitamos y con urgencia la intervención estatal, para garantizar escuelas, colegios, universidades de calidad, con profesionales ecuatorianos en la dirección y docencia, seleccionados en base a sus méritos, sin injerencias políticas o partidistas.

Ya en la etapa universitaria, el rol que cumplen las facultades y escuelas de derecho es fundamental, pues sus propuestas deben consistir en programas académicos de excelencia cuidadosamente diseñados y dirigidos a la formación de profesionales con las competencias necesarias para enfrentar los retos de una sociedad anhelante de justicia.

En éste contexto, las mallas curriculares deben enfocarse a la formación profesional básica e integral, basada en principios jurídicos, combinando durante sus años de estudioaprendizaje la teoría con la práctica, de tal forma que el estudiante pueda tener un acercamiento real a lo que va a ser el ejercicio de su profesión, sometiendo a sus alumnos a rigorosas pruebas de suficiencia académica; motivando además a no conformarse con los conocimientos adquiridos en la universidad, sino a su auto preparación, a incursionar en la investigación y vinculación comunitaria; y, que una vez obtenido su título profesional busquen su perfeccionamiento - especialización, a través de posgrados de calidad (maestrías y doctorados), al nivel de las grandes universidades mundiales, ofertados por universidades públicas y privadas ecuatorianas, en base a la demanda laboral, y con igualdad de oportunidades, hombres- mujeres, jóvenes - adultos, sin distinción de clases; y de esa forma hacer efectivo nuestro derecho a la educación, encontrando oportunidades académicas y laborales en nuestro propio país, sin tener que dejar nuestras familias, trabajos; ofreciendo nuestro talento, capacidad y aporte económico de preparación al servicio de la patria.

Siempre se ha escuchado que lo importante no es la cantidad, sino la calidad y ejercicio propositivo al servicio de la comunidad; es por ello que se deberían conocer y revisar las estadísticas que influyen al Estado actualmente a discriminar la formación de nuevos abogados, pues si bien un abogado generalista ya no tiene asidero en una sociedad como la nuestra, precisamente por la multiplicidad de oportunidades de desarrollo en nuevas áreas relacionadas al cambio de la matriz productiva ecuatoriana, como la minería responsable, los conflictos, la producción energética alternativa, los nuevos parámetros de políticas públicas con más competencias para las unidades pequeñas como las juntas parroquiales, la incursión en la tecnología aeroespacial, etc., se requieren de jurisconsultos especializados en cada una de estas áreas. Lo trascendental entonces, es la calidad, la formación académica y honestidad en el ejercicio de su profesión, no podemos estar frente a una miopía estatal, de limitación de carreras, pues en el caso de la abogacía, el campo profesional es amplio, la incidencia de los abogados se evidencia en cada actividad del ser humano; de ahí, que las especializaciones, maestrías, doctorados a impartirse en el Ecuador, sean coordinadas en base a las líneas de desarrollo estatales.

El ejercicio honesto de nuestra profesión, nos posibilita ser responsables en las soluciones estatales y constructores de éste gran país, que hoy más que nunca requiere la presencia y trabajo sacrificado de sus hijos; necesitamos profesionales especializados, docentes, funcionarios de estado y autoridades de elección popular capacitados, preparados para su desempeño, con maestrías y doctorados, como política de Estado.

En este estado de cosas adquiere singular importancia la identificación irrestricta del estudiante o del profesional en Derecho con aquellos altos ideales que promovieron la búsqueda existencial relacionada hacia el ejercicio de una profesión en particular. 
La formación del abogado en el Ecuador requiere la generación de espacios propicios para el debate sobre la pertinencia y legitimidad de medidas restrictivas para el libre acceso a ésta profesión, ya hemos aportado datos básicos, respecto a la visión que tiene la autora, sobre un tema en el que estamos inmersos algunas escuelas de derecho en nuestro territorio, que si bien nacen de una visión particular sobre el orgullo que representa, en éste análisis crítico, el hecho de percibir la importancia que tiene el ejercicio responsable y ético de nuestra profesión como propulsor de los motores de desarrollo que de manera reiterativa plantea la institucionalidad actual en su discurso sobre el tema.

De esta manera somos sinceros al plantear desde el ejercicio de la docencia una convocatoria renovada a las nuevas generaciones a no abdicar a su proyecto de vida, si es que éste sigue siendo un llamado a ejercer la abogacía en el Ecuador contemporáneo. 


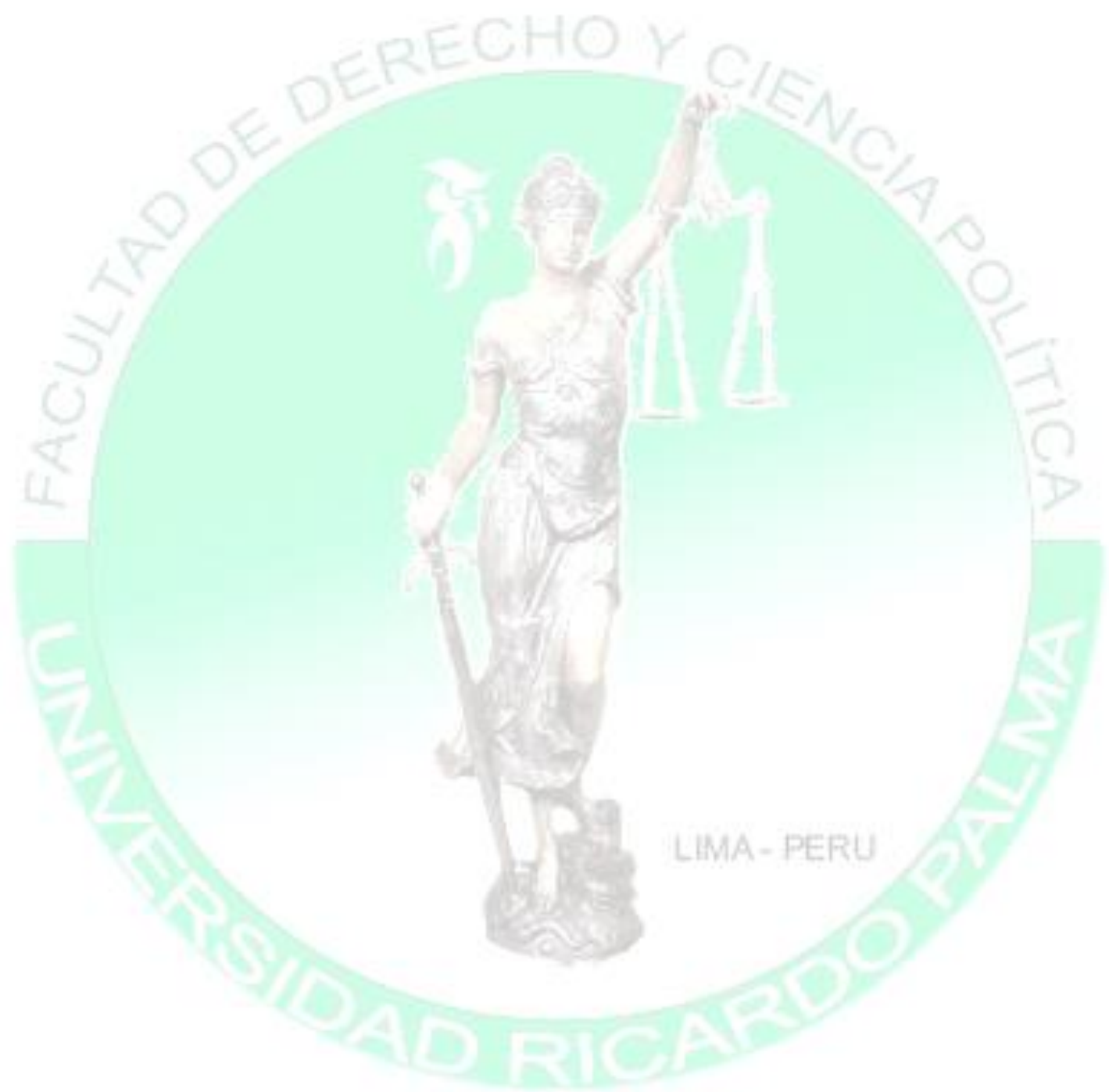

